\title{
NAD(P)H Fluorescence Lifetime Imaging of Live Intestinal Nematodes Reveals Metabolic Crosstalk Between Parasite and Host
}

\section{Wjatscheslaw Liublin}

German Rheumatism Research Centre

\section{Sebastian Rausch}

Freie Universität Berlin

\section{Ruth Leben}

German Rheumatism Research Centre

Juliane Liebeskind

Charité - Universitätsmedizin, Humboldt University

Anja E. Hauser

Charité - Universitätsmedizin, Humboldt University

\section{Susanne Hartmann}

Freie Universität Berlin

Raluca A. Niesner ( $\square$ raluca.niesner@fu-berlin.de )

German Rheumatism Research Centre

Ingeborg Beckers

Berliner Hochschule für Technik

\section{Research Article}

Keywords: intestinal nematodes, ambivalent impact, immunomodulatory, parasites, metabolism, NADPH

Posted Date: September 17th, 2021

DOI: https://doi.org/10.21203/rs.3.rs-881040/v1

License: (1) (1) This work is licensed under a Creative Commons Attribution 4.0 International License. Read Full License 


\section{Abstract}

Infections with intestinal nematodes have an ambivalent impact: they represent a burden for human health and animal husbandry, but, at the same time, may ameliorate auto-immune diseases due to the immunomodulatory effect of the parasites. Thus, it is key to understand how intestinal nematodes arrive and persist in their luminal niche and interact with the host over long periods of time. The basic mechanism ruling over parasite and host cellular and tissue functions, the metabolism, was largely neglected in the study of intestinal nematode infections. Here we use NADH (nicotinamide adenine dinucleotide) and NADPH (nicotinamide adenine dinucleotide phosphate) fluorescence lifetime imaging of explanted murine duodenum infected with the natural nematode Heligmosomoides polygyrus and define the link between general metabolic and specific enzymatic activity in parasite and host tissue, during acute infection. In both healthy and infected host intestine, energy is effectively produced, mainly via oxidative phosphorylation. In contrast, the nematodes shift their energy production from balanced fast anaerobic glycolysis and effective oxidative phosphorylation, towards mainly anaerobic glycolysis, back to oxidative phosphorylation during the different life cycle phases in the submucosa versus the intestinal lumen. Additionally, we found an increased NADPH oxidase (NOX) enzymes-dependent oxidative burst in infected intestinal host tissue as compared to healthy tissue, which was mirrored by a similar defense reaction in the parasites. We expect that, the here presented application of in vivo $\mathrm{NAD}(\mathrm{P}) \mathrm{H}$-FLIM constitutes a unique tool to study metabolic host-parasite crosstalk, in various parasitic intestinal infections.

\section{Introduction}

Infections with gastrointestinal nematodes constitute a central burden for the global health of humans and animals alike. Approximately $25 \%$ of the world's population is affected, especially in the tropical and subtropical regions. In animal husbandry, nematode infections are widely distributed and lead to huge economic losses in farming. Heligmosomoides polygyrus is a natural parasite of the gastrointestinal tract of mice and a widely used model to study intestinal nematode infections. When $H$. polygyrus larvae are taken up orally, they invade the intestinal submucosa, develop to L4 larvae and further into adult worms, which reenter the intestinal lumen. The adult worms move to the proximal part of the small intestine, where they persist and induce chronic infection. Whereas the modulatory effects of the nematode infection on the host immune system and on the microbial environment have been investigated ${ }^{1-8}$, less it is known about the enzymatic activity, energetics and metabolism of the nematodes themselves, which rule over the way the parasites persist in the intestinal environment. Up to now, information on metabolism of live parasites (e.g. nematodes) in the host intestine was mostly acquired using bulk biochemical approaches, without spatial or temporal specification ${ }^{9}$.

Intravital two-photon microscopy extended by fluorescence lifetime imaging (FLIM) of nicotinamide adenine dinucleotide (NADH) and nicotinamide adenine dinucleotide phosphate (NADPH), hereafter $\mathrm{NAD}(\mathrm{P}) \mathrm{H}$, provides one of the most versatile tools to investigate $\mathrm{NAD}(\mathrm{P}) \mathrm{H}$-dependent metabolic activity in live tissues, with cellular or even subcellular resolution ${ }^{10-12}$. 
Two-photon laser-scanning microscopy ${ }^{13}$, characterized by large imaging depth and optical sectioning in the tissues of living animals, provides information on cellular dynamics and orchestration during diverse pathophysiological situations. In order to account for cellular and tissue functionality, in vivo metabolic imaging, is required for a full understanding of these pathophysiological phenomena, besides the analysis of cellular dynamics and communication. This has previously been achieved by combining NAD (P)H FLIM with two-photon microscopy in mammals, especially in mice ${ }^{14}$. However, there is only a single study using the free-living nematode Caenorhabditis elegans by means of NAD(P)H-FLIM showing specific metabolic pathways in various tissue regions of the worm, associated with the respective cellular differentiation stage $\mathrm{e}^{15}$. The genuine environment of $H$. polygyrus is the murine gut, in contrast to $C$. elegans, which lives in the soil. In order to characterize the metabolism of $H$. polygyrus, ruling over locomotion, persistence and defense in the gut of the host, we aimed to establish NAD(P)H-FLIM of the parasite in the murine gut.

The fluorescence lifetime gives information about the molecular environment of the investigated fluorophore. As such, fluorescence lifetime imaging has been used to quantify different vital parameters in cells and tissues, e.g. ionic strength, calcium levels, $\mathrm{pH}$ values, protein folding and cleavage using Foerster Resonant Energy Transfer (FRET), temperature or viscosity ${ }^{16-29}$. The fluorescence of the ubiquitous co-enzymes NADH and NADPH has been extensively used for FLIM to monitor metabolic activity ${ }^{27}$. The $\mathrm{NAD}(\mathrm{P}) \mathrm{H}$ metabolic activity can be correlated to specific cellular phenotypes and functions, with application in health and different pathologies ${ }^{14}$. While many technologies to perform FLIM have been proposed, both in time and in frequency domain ${ }^{30-33}$, time-correlated single-photon counting proved to be most reliable and found broad application for deep-tissue imaging.

In the current work, we used time-correlated single photon counting by two-photon laser-scanning microscopy to demonstrate the feasibility and benefits of NAD(P)H-FLIM to monitor specific metabolic pathways of the host tissue and, in parallel, of the intestinal parasite H. polygyrus. By applying our systematic evaluation of NAD(P)H-FLIM data, we quantified the NAD(P)H-dependent enzymatic and general metabolic activity of both the host and the parasite and thereby received insights into the hostparasite crosstalk.

\section{Results}

\section{$\mathrm{NAD}(\mathrm{P}) \mathrm{H}$ fluorescence lifetime imaging of murine intestinal environment}

Using NAD $(\mathrm{P}) \mathrm{H}$-FLIM, we analyzed the general metabolic activity and specific enzymatic activity of the nematode $H$. polygyrus and of its natural environment, the luminal side of duodenum in mice. In order to access the intestinal niche of adult H. polygyrus nematodes, duodenum of infected C57/BI6 mice was explanted and cut open to expose its luminal side. Here, the parasites, coiled around intestinal villi, persist over several weeks (Fig. 1a). 
The fluorescence of the ubiquitous coenzymes $\mathrm{NAD}(\mathrm{P}) \mathrm{H}$ was detected at $466 \pm 30 \mathrm{~nm}$ after two-photon excitation at $760 \mathrm{~nm}$. The coenzymes present both in nematodes and in enterocytes and cells of the lamina propria of murine intestinal villi, were also used to visualize worm and host tissue architecture. Based on $\mathrm{NAD}(\mathrm{P}) \mathrm{H}$ fluorescence images, we used a UNet-based pixel classification algorithm to segment the nematodes as well as epithelial layer and lamina propria of the murine intestinal villi and to exclude background regions (Fig. 1b; Material and Methods).

The time-resolved $\mathrm{NAD}(\mathrm{P}) \mathrm{H}$ fluorescence decay acquired in each pixel of the image (Fig. 1c) was recorded by time-correlated single-photon-counting ${ }^{34,35}$ and evaluated using the phasor approach to $\mathrm{FLIM}^{36}$, generating the real and imaginary phase maps and the average fluorescence lifetime (t) map (Fig. 1c).

The time-resolved $\mathrm{NAD}(\mathrm{P}) \mathrm{H}$ fluorescence signal provided further information on the molecular environment in cells and tissue. The coenzymes NADH and NADPH are able to bind to diverse enzyme and participate in this way to different metabolic pathways and to reductive biosynthesis within cells ${ }^{29,37-}$ 40. The fluorescence lifetimes of both unbound NADH and unbound NADPH lay at $\approx 450 \mathrm{ps}^{41,42}$, both being the average of the fluorescence lifetime over two folding states of each type of coenzyme molecule $(\approx 200 \mathrm{ps} \text { and } \approx 700 \mathrm{ps})^{43}$. When bound to enzymes, the fluorescence lifetime of $\mathrm{NAD}(\mathrm{P}) \mathrm{H}$ becomes longer $(\approx 2000 \mathrm{ps})$, and it strongly depends on its enzymatic binding site. Taking into account the abundance of NAD $(P) H$-dependent enzymes in living cells (mostly valid across species), we previously developed a systematic frame work to evaluate NAD (P)H-FLIM data ${ }^{44}$. This approach allows us to assess the general metabolic activity, i.e. the percentage of bound $N A D(P) H$ from the total $N A D(P) H$ - activity map and the preferential specific enzymatic activity - enzyme map - in each pixel of the acquired image, both for the worm and for the host tissue (Fig. 1d). High values in the activity map indicate high metabolic activity, whereas low values indicate decreased metabolism, possibly associated with dormancy, low temperature or cell death ${ }^{45}$. The preferential binding of $\mathrm{NAD}(\mathrm{P}) \mathrm{H}$ to specific enzymes in the enzyme map indicates various metabolic pathways within cells and tissues, e.g. a preferential activation of lactate dehydrogenase (LDH) is associated with anaerobic glycolysis. The activation of pyruvate dehydrogenase (PDH) is associated with oxidative phosphorylation, while the activation of NADPH oxidases (NOX/DUOX enzymes) indicates metabolic defense, i.e. via oxidative burst. Thus, the heterogeneity of dominant metabolic pathways in worm and host, with high axial $(\mathrm{z})$ resolution is monitored (Fig. 1e).

\section{$\mathrm{NAD}(\mathrm{P}) \mathrm{H}$ metabolism of explanted duodenum}

By performing NAD(P)H-FLIM in healthy, explanted duodenum (Fig. 2a) of C57/BI6 mice, in RPMI medium, at $37^{\circ} \mathrm{C}$, we assessed the metabolic activity of intestinal epithelial cells (EC) and of cells within the lamina propria $(L P)$, respectively, as segmented from 3D NAD $(P) H$ fluorescence images using the previously described pixel classification algorithm (Fig. 2b,c).

During imaging over up to 3 hours after explanting the duodenum, the general metabolic activity of the EC was $78.4 \pm 5.5 \%$ ( $n=6$ mice, field-of-view $500 \times 500 \mu m^{3}$ over 50 to $110 \mu \mathrm{m}$ depth). In some mice, the 
cells in lamina propria show a slightly lower metabolic activity as compared to EC, in most mice the activity levels were comparable for lamina propria and epithelium, resulting into an activity value of $76.3 \pm 5.2 \%$ in LP $(p=0.5139)$. The variability of metabolic activity was slightly higher in lamina propria than in the epithelium, i.e. standard deviation of activity levels per mouse were $8.1 \%$ in LP as compared to $6.3 \%$ in EC. These results resemble our findings regarding metabolic activity in small intestine villi of anesthetized $C X_{3} C R 1$ :GFP mice acquired using in vivo NAD(P)H-FLIM ${ }^{12}$.

The enzymatic activity profile in the explanted healthy duodenum shows a preference towards oxidative phosphorylation in epithelium, with local increased activation of NADPH oxidases (Fig. 2d,f). As the first line of cellular defense in the intestine, EC show a high expression of NOX4 and NOX2 ${ }^{12}$. Both NOX2 and NOX4 activation leads to oxidative burst, i.e. massive extracellular reactive oxygen species (ROS) generation needed in the defense against pathogens. In addition, NOX4 activation is used for intercellular communication among intestinal epithelial cells ${ }^{46}$, in support of host defense. In contrast, the cells in LP balance anaerobic glycolysis and oxidative phosphorylation for energy production, with a slight preference towards anaerobic glycolysis. The LP cells show only few isolated spots of NADPH oxidase(s) activation, presumably NOX2 in phagocytes, significantly lower than the NOX activation observed in epithelium (Fig. 2e,f). These data are also in good agreement with the findings of analogous intravital experiments ${ }^{12}$.

\section{Changes in metabolic activity in the intestinal villi during $H$. polygyrus infection}

$\mathrm{NAD}(\mathrm{P}) \mathrm{H}$ fluorescence images of duodenum tissue from mice infected with $H$. polygyrus revealed a disorganization of the typical villi architecture in the vicinity of nematodes (Fig. 3a, Suppl. Fig. 1). Consequently, segmenting epithelium and lamina propria, as described for the healthy duodenum, was not always possible in the infected gut and we refer in all following data to intestinal tissue, without differentiating between epithelium and lamina propria.

When compared to healthy duodenum (77.3 $\pm 5.2 \%), \mathrm{NAD}(\mathrm{P}) \mathrm{H}-\mathrm{FLIM}$ analysis of infected duodenum tissue, 6 to 14 days post infection (acute infection), revealed a similarly high metabolic activity $(74.0 \pm 7.6$ $\%, \mathrm{n}=9$ mice, Fig. 3c,d), with high heterogeneity within the villi (s.d. $9.6 \%)$. A preference towards oxidative phosphorylation was observed in all mice (Fig. 3b,e,f). In contrast, at an earlier time point of infection, at day 6 after infection, where most parasites still reside in the submucosa, the typical villus morphology is preserved. At this time point during infection, in some areas in one mouse, a preference towards anaerobe glycolysis was observed (Fig. 3b, left image), however, in most duodenal villi, oxidative phosphorylation prevailed ( $n=2$ mice, Fig. $3 e, f)$.

In line with the expected time course of immunomodulation caused by nematodes in the host $3,5,8,47-50$, NADPH oxidases activation in the villi is rather low, i.e. comparable to healthy tissue, at day 6 after infection but increases dramatically at day 10 and 14 after infection, i.e. during the acute phase, in the vicinity of parasites (Fig. 3e,f). 


\section{NAD(P)H metabolic and enzymatic activity of live $H$. polygyrus in the gut}

Adult H. polygyrus nematodes residing in the intestinal lumen display active motility of loose ends of their body, i.e. the ends not being coiled around villi (Fig. 4a, Suppl. Movie 1, 2). This active, undulatory motion through the intestinal mucus (labelled by the lipophilic dye BODIPY1) indicates the vitality of the worms and probably is key for their locomotion upwards in the small intestine and their survival in the duodenal luminal niche. In order to understand the energetics governing their motility behavior, we aimed to investigate $\mathrm{NAD}(\mathrm{P}) \mathrm{H}$ metabolism of nematodes by $\mathrm{NAD}(\mathrm{P}) \mathrm{H}-\mathrm{FLIM}$.

Using the same pixel classification approach as previously described, the nematode hydrostatic skeleton and its cellular, $\mathrm{NAD}(\mathrm{P}) \mathrm{H}$-rich tissue were segmented from the $\mathrm{NAD}(\mathrm{P}) \mathrm{H}$ intensity images of $H$. polygyrus in the murine duodenum (Fig. $4 \mathrm{~b}$ ). The resulting mask was used to generate metabolic and enzyme 2D and 3D maps of the worms (Fig. 4c, d) and to calculate the relative preferential activation of the different metabolic pathways (Fig. 4e). Interestingly, several worms showed extensive NADPH oxidase activation, presumably DUOX2 activation, as the only member of the NADPH oxidases family expressed in $H$. polygyrus ${ }^{51}$. The activation of DUOX2 was particularly high in the digestive system and may indicate parasite defense against nematode environment.

\section{Metabolic and specific enzymatic activity of $H$. polygyrus depends on the specific phase during infection}

In order to understand if the translocation from the larval development in the submucosa to an adult worm in the gut lumen is associated with a specific metabolic pathway, we tracked the changes in $\mathrm{NAD}(\mathrm{P}) \mathrm{H}$ metabolism of $H$. polygyrus parasites between day 6 and day 14 after infection, using $\mathrm{NAD}(\mathrm{P}) \mathrm{H}-$ FLIM (Fig 5a, b, c).

As compared to the host intestinal tissue, nematodes show a lower metabolic activity $(64.3 \pm 6.3 \%, n=16$ worms from 14 mice, between day 6 to 14 after infection), with a similarly high heterogeneity per worm of $9.3 \%$. Interestingly, the general metabolic activity in worms slightly increases from day 6 to day 14 after infection, i.e. day 6 metabolic activity $58.6 \pm 2.2 \%$ ( 2 worms), heterogeneity per single worm $6.1 \%$, day 10 $61.1 \pm 9.9 \%$ and $11.8 \%$ (5 worms), day $1264.5 \pm 5.8 \%$ (4 worms) and day $1473.1 \pm 5.9 \%$ (5 worms) (Fig. $5 d)$.

At day 6 after infection, $H$. polygyrus parasites are located in the intestinal submucosa. At this stage, they generate energy both by anaerobe glycolysis and by oxidative phosphorylation and show very low levels of oxidative burst (DUOX2 activation). 10 days after infection, the parasites start to emerge from the submucosa into the lumen. This process is associated with a metabolic shift towards anaerobe glycolysis, at still low DUOX2 activation. Between day 12 and day 14 after infection, the adult parasites shift their metabolism towards oxidative phosphorylation and show increased levels of DUOX2 activation (oxidative burst), indicating defense mechanisms, possibly against the host (Fig. 5e,f).

\section{Discussion}


Understanding physiological and pathological phenomena require the spatio-temporal analysis of cell phenotypes and functions in their genuine environment, the living tissue or even living organism. Due to reduced scattering of the excitation wavelengths and tissue-friendly confinement of excitation around the focal point of the objective lens, two-photon fluorescence laser-scanning microscopy ${ }^{13}$, and more recently, three-photon fluorescence laser-scanning microscopy ${ }^{52}$ have proven to be the most versatile tools fulfilling this requirement. In the past decades, these technologies brought new insights about cellular dynamics and communication both in health and in disease, particularly in neurosciences ${ }^{53-55}$, immunology ${ }^{56-60}$ and oncology ${ }^{61-63}$.

Additionally, extending two-photon microscopy with fluorescence lifetime imaging (FLIM) ${ }^{27}$ allowed access to quantitative information regarding cellular and tissue functions in vivo. For instance, FLIM has been used in combination with FRET-based calcium constructs expressed in transgenic mice to monitor absolute calcium levels in a cell-specific manner ${ }^{64,65}$. In order to monitor cellular metabolism, being the cornerstone of cellular and tissue function, in vivo FLIM of the ubiquitous metabolic co-enzymes nicotinamide adenine dinucleotide (NADH) and nicotinamide adenine dinucleotide phosphate (NADPH) has been performed. This retrieves both the general metabolic activity of cells as well as information on specific enzymes, which preferentially bind to $N A D(P) H$, i.e. participate in biochemical reactions. In this way, the $N A D(P) H$-dependent metabolism could be cross-linked to cellular vitality / dormancy ${ }^{35}$ and cellular differentiation, under physiologic conditions ${ }^{17}$ and in tumors ${ }^{39}$. Additionally, it could be related to (regular or aberrant) defense mechanisms in various tissues ${ }^{10,11,45}$. In order to extend the access to information contained in NAD(P)H-FLIM data, a systematic evaluation frame-work of these data has been proposed ${ }^{44}$, which includes the fluorescence lifetimes of $\mathrm{NAD}(\mathrm{P}) \mathrm{H}$ bound to the most abundant $\mathrm{NAD}(\mathrm{P}) \mathrm{H}-$ dependent enzymes across species.

Whereas, up to now, several studies employed in vivo NAD(P)H-FLIM to investigate metabolism in mammals, especially in mice, there are no in vivo studies concerning the NAD $(P) H$-dependent metabolism of intestinal pathogens such as nematodes and its cross-link to the metabolism of the host tissues.

By using NAD(P)H-FLIM upon two-photon excitation at $760 \mathrm{~nm}$, we studied the metabolism of explanted intestinal duodenal tissue and, in parallel, of the parasitic worm Heligmosomoides polygyrus, in wild type C57/BI6 mice. We found that the metabolic activity patterns of the intestinal villi, indicative for metabolic fitness, in explanted duodenum are comparable to intestinal tissue imaged under intravital conditions ${ }^{12}$. Besides, a similar pattern of NADPH oxidases activation, i.e. higher in epithelial cells than in cells of the lamina propria, was detected both in explanted duodenum, as presented here, and in the small intestine of living mice ${ }^{12}$. In this way, we validated the experimental model for analyzing the cross-link between host and parasite, along the metabolic axis.

With regard to the metabolic activity of the host tissue during the presence of nematodes in the intestinal lumen, during the acute phase of the infection, we revealed a strong defense reaction by the host, in the direct vicinity of parasites. This effect was not observed in the villi before adult worms reemerge from the 
submucosa, where invading stage 3 larvae develop, via an L4 larval stage, into adults. This defense reaction in the intestinal lumen indicated by a strong activation of NADPH oxidases in epithelium and in the lamina propria was associated with a disintegration of the typical tissue architecture of the villi, in direct nematode vicinity. This finding is in line with a strong anti-nematode host immune response associated with GATA-3 + Th2 cells and GATA-3 + T-bet + Th2/ 1 hybrid cells, production of the respective cytokines (IL-4, IL-5, IL-13, IFN-g) as well as a prominent eosinophilia at the side of infection ${ }^{1,66}$. Thus, during the acute phase of infection, the strong activation of NADPH oxidase and tissue disintegration coheres with a prominent anti-pathogen immune response. A silencing of the host defense, in line with the shift towards an immunoregulatory response suppressing the Th2 activity ${ }^{1}$, is expected during chronicity of infection - a stage not monitored in this study.

Deciphering the metabolic activity of the parasite paralleled their motility level and, possibly, their differentiation stage. $H$. polygyrus parasites balanced energetic metabolism of effective oxPhos (associated with low motility) and of energy-demanding anaerobe glycolysis (associated with either maturation or active movement), when residing in the submucosa. Our data show that, when the nematodes emerge from the submucosa into the intestinal lumen, they shift their metabolism towards mainly anaerobe glycolysis for rapid energy production. After arriving in their persistence niche in the duodenum, they shift back to effective energy production, i.e. mainly oxPhos.

DUOX2 - the only member of the NADPH oxidases family expressed in H. polygyrus ${ }^{51}$ - is highly activated in the gastrointestinal system and partially in the cuticle of parasites residing in the duodenal persistence niche. In contrast, parasites in the submucosa do not show DUOX2 activation. Presumably, due to the molting process of the parasite from larval stage 3 via larval stage 4 into adults which is associated with stage-specific parasite antigens. During this process, there is probably no need to invest additional energy to defend themselves via oxidative burst. DUOX2 activation in parasites increases during the course of infection up to day 14 , when emerging into the intestinal lumen. The presence of DUOX1/2 was previously reported in cuticle and intestine of $C$. elegans ${ }^{67}$, a soil nematode, in line with our observations regarding the location of activated DUOX2 in H. polygyrus.

In conclusion, the here presented in vivo $\mathrm{NAD}(\mathrm{P}) \mathrm{H}-\mathrm{FLIM}$ approach to study metabolism of intestinal nematodes in a genuine tissue context provides us with the unique opportunity to decipher a new dimension of the host-parasite crosstalk. In future, this will be relevant for multiple issues such as the investigation of detrimental effects of the long-term chronic phase of the parasitic infection, a more efficient exploitation of nematode infections in the immunomodulatory treatment of autoimmune diseases, or for studying the interaction of the intestinal nematodes with gut microbes as well as concurrent co-infecting gut pathogens.

\section{Material And Methods}

\section{Two-photon laser-scanning microscope setup for fluorescence lifetime imaging of $N A D(P) H$}


Two-photon fluorescence lifetime imaging experiments were performed using a specialized laserscanning microscope based on a commercial scan head (TriMScope II, LaVision Biotec - a Miltenyi company, Bielefeld, Germany). A near-infrared laser (Ti:Sa, Chameleon Ultra II, Coherent, Duisburg, Germany) tuned at $760 \mathrm{~nm}$, repetition rate $80 \mathrm{MHz}$, and pulse width $140 \mathrm{fs}$ was used as excitation source for $\mathrm{NAD}(\mathrm{P}) \mathrm{H}$. The linearly polarized Ti:Sa beam was scanned over the sample by galvanometric mirrors. A water-immersion objective lens (20x, NA 1.05, Apochromat, Olympus, Hamburg Germany) was used to focus the laser beam into the explanted duodenum samples. The laser power was controlled by a combination of $\lambda / 2$ wave-plates and polarizers. The ultrashort pulses of the laser were compressed using an external compressor. NADH and NADPH fluorescence was collected in the backward direction using a dichroic mirror (775, Chroma, Marlborough, MA, USA), passed through an interference filter (466 $\pm 30 \mathrm{~nm}$ ) and was detected by a GaAsP PMT (Hamamatsu, Herrsching, Germany) connected to the previously described TCSPC electronics ${ }^{31}$ (LaVision Biotec - a Miltenyi company, Bielefeld, Germany). The TCSPC data were collected at a time resolution of 55 ps, over at least 9 ns and with a Gaussian-shaped instrument response function of 250 ps FWHM. In all imaging experiments, we used an average maximum laser power of $30 \mathrm{~mW}$ to avoid photodamage. The acquisition time for an image with a fieldof-view of $500 \mu \mathrm{m} \times 500 \mu \mathrm{m}$ and a digital resolution of $505 \times 505$ pixel was $800 \mathrm{~ms}$.

\section{Phasor analysis of time-domain FLIM data}

Fluorescence lifetime data were analyzed as previously described ${ }^{44}$. The phasor approach transforms the time-domain data (the fluorescence decay), into a virtual, normalized phase domain by calculating the discrete Fourier transformation numerically (modulations frequency $=80 \mathrm{MHz}$ ). The transformation leads to a complex number, the real and imaginary parts of which give the coordinates of the vector in the phase domain ("phasor"). That vector originated in (0|0) and points towards the half circle (centrum at $(0.5 \mid 0)$, radius $=0.5)$, due to the exponential nature of the original time domain data. Because of the value normalization, the real part of the phasor reaches from 0 to 1 , and those of the imaginary part from 0 to 0.5. In this way, short fluorescence lifetimes of homogeneous fluorophores (mono-exponential decay) are located on the half circle at large real values, whereas with increasing lifetime, the real value decreases.

As previously described ${ }^{44}$, the general metabolic activity (displayed in the activity map) is geometrically calculated as the distance from each experimental data point in the phasor plot to the intersection of the segment connecting the data point and the predefined position of free $\mathrm{NAD}(\mathrm{P}) \mathrm{H}$ lifetime with the half circle. Since this distance relates to the total length of the segment, from the free $N A D(P) H$ position to the second intersection of the line with the half circle (representing fully enzyme-bound $N A D(P) H)$, the activity is expressed in percentage: $0 \%$ only free $N A D(P) H, 100 \%$ only enzyme-bound $N A D(P) H$.

Moreover, the enzymatic activity (enzyme map) displays in each pixel the probability that $N A D(P) h$ is bound to a specific enzyme. The enzyme was selected based on a similarity approach, comparing the angles between the predefined segments connecting the free $\mathrm{NAD}(\mathrm{P}) \mathrm{H}$ position and the specific enzymebound $N A D(P) H$ positions with the line connecting each experimental data point with the free $N A D(P) H$ position. 


\section{Mice and infection model}

All mice used were C57BI/6 mice. 200 stage 3 (L3) H. polygyrus larvae were given orally to female 10-12 weeks old mice, as previously described ${ }^{1}$. Imaging experiments were performed during the acute phase of the infection between day 6 and day 14 after infection.

All animal experiments were performed in accordance with the National Animal Protection Guidelines and approved by the Berlin Ethics Committee for the Protection of Animals (G0176/16).

All animal experiments were performed in accordance with the ARRIVE guidelines. The present study compares the duodenum of healthy C57/BI6 mice (control group) with those of mice infected with the intestinal nematode $H$. Polygyrus, during the acute phase of infection, day 6 to day 14 after infection. In the group of infected mice, we performed a further stratification of the results referring to the time point after infection (at day 6 , day 10 and day 14). $\mathrm{n}$ refers to number of animals, with 20 to 50 tissue layers from a $1 \mathrm{~cm}$ piece of duodenum in each animal. In the control group, we included 7 mice and in the group of infected mice we included 9 mice. We omitted a calculation of sample size due to the exploratory character of the present study. Due to the same reason, we performed no randomization and no blinding.

\section{Preparation of the freshly explanted duodenum samples for imaging}

Mice were sacrificed by cervical dislocation. The small intestine was freshly explanted, the duodenum was cut of the rest of the small intestine, and placed on ice. After opening up the duodenum tube to expose the lumen with adult parasites, the duodenum was fixed on a Petri dish using tissue glue and immersed in RPMI medium containing $10 \%$ FCS. The Petri dish was placed on the microscope table for two-photon imaging - maximally 3 hours after explanting. A temperature of $37^{\circ} \mathrm{C}$ was maintained at all times during the experiment using a heating foil. In some experiments, the mucus was labelled by BODIPY1 (Thermo Fisher, Bremen, Germany).

\section{Data analysis}

By summing up the intensity values of the acquired time-resolved $\mathrm{NAD}(\mathrm{P}) \mathrm{H}$ fluorescence images, the phasor-analysis Python routine provides an intensity image for each depth in tissue, containing only spatial information. The resulting z-stacks are typically $505 x 505 x \approx 80$ voxel. We first individually normalized the contrast and brightness of all depth slices within a z-stack using a dedicated Fiji-macro. For segmentation of different tissue areas, we used the 'Pixel-Classification' function in ILASTIK. Thereby, we created neural networks with adapted parameters for all conditions. In detail, we manually assigned the pixels of about one third of the slices in the z-stack and used these for training the neural network. The slices were evenly distributed over the entire depth of the stack. Another third of the slices were used for validation. Feature selection included intensity, edges, texture and structure with weights increasing in this order and with ALPHA values between 0.3 and 100. Training data was labeled with a maximum of three classes: (i) for healthy murine intestinal tissue: background, epithelium and lamina propria, (ii) for infected tissue: background, host intestinal tissue and worm and (iii) for segmented parasites: 
background, hydro-skeleton and enzyme-rich tissue. The masks generated by ILASTIK were applied to the enzyme maps, activity maps and intensity projections. Subsequently, we weighted the in the preprocess Gaussian-filter-smoothed enzyme and activity maps with the segmented intensity images using a Fiji macro and assigned a LUT according to the pixel values (enzyme-map 1-13, activity-map 0-100\%). The sum of each enzyme for each masked slice of a measurement was counted and stored in a text file. From this, we generated the $z$-abundance plots with (enzymes $x$ stack-depth $x$ abundance as pixel intensity). Correspondingly, the mean value and the standard deviation of the activity of each slice of the measurement was calculated. Using Matplotlib and numPy in a Python script, we then created box-plots for the enzyme-frequency plots from the text-stored-values over the depth for each measurement or measurement series.

Using the enzyme activity abundance information in each depth of a z-stack - either for the murine tissue or for the enzyme-rich tissue of the nematodes, we calculated the relative occurrence of anaerobe glycolysis, i.e. the sum of the abundance of the enzymes grouped around LDH, as compared to that of oxPhos (aerobe glycolysis), i.e. the sum of the abundance of the enzymes grouped around PDH, and to the oxidative burst, i.e. abundance of NOX and DUOX enzymes.

For statistical analysis, we used ANOVA tests (ns $p>0.05,{ }^{*} p<0.05,{ }^{* \star} p<0.01,{ }^{* \star *} p<0.001$ ). In case of multi-column comparison, we used the Bonferroni test. All data in the manuscript are presented as mean standard \pm deviation.

\section{Declarations}

\section{Acknowledgements}

We thank Robert Günther for excellent technical support. Financial support from the German Research Council (DFG) under grants TRR130, C01 and SFB1444, P14 (R.A.N., A.E.H.), HA5354/8-2 (A.E.H.) and GRK2046 B4 and B5 (S.H., S.R.) and HA 2542-/8-1 (S.H.) are greatly acknowledged. W.L. receives a PhD fellowship form the Berliner Hochschule für Technik, School of Applied Sciences, Berlin in Medical Physics / Physical Engineering.

\section{Authors' contribution}

W.L. has performed experiments, analyzed data, wrote manuscript. R.A.N. and S.H. conceived the study, analyzed and interpreted data, wrote the manuscript. A.E.H. contributed with expertise regarding live intestinal tissue imaging and edited the manuscript. W.L., I.B. and R.L. developed and adapted the algorithm for FLIM data analysis. S.R. and J.L. performed experiments. S.R. wrote manuscript.

\section{Conflict of interests}

We declare no conflict of interests.

\section{Ethical approval}


All animal experiments were performed in accordance with the National Animal Protection Guidelines and approved by the Berlin Ethics Committee for the Protection of Animals (G0176/16) as part of the

Landesamt fuer Gesundheit und Soziales (LaGeSo), Berlin (the State Office for Health and Social Affairs).

\section{References}

1. Affinass, N., Zhang, H., Lohning, M., Hartmann, S. \& Rausch, S. Manipulation of the balance between Th2 and Th2/1 hybrid cells affects parasite nematode fitness in mice. Eur $J$ Immunol 48, 1958-1964, doi:10.1002/eji.201847639 (2018).

2. Hartmann, S. et al. Gastrointestinal nematode infection interferes with experimental allergic airway inflammation but not atopic dermatitis. Clin Exp Allergy 39, 1585-1596, doi:10.1111/j.13652222.2009.03290.x (2009).

3. Hepworth, M. R. et al. Mast cells orchestrate type 2 immunity to helminths through regulation of tissue-derived cytokines. Proc Natl Acad Sci U S A 109, 6644-6649, doi:10.1073/pnas.1112268109 (2012).

4. Rausch, S. et al. Establishment of nematode infection despite increased Th2 responses and immunopathology after selective depletion of Foxp3+ cells. Eur J Immuno/ 39, 3066-3077, doi:10.1002/eji.200939644 (2009).

5. Rausch, S. et al. Parasitic Nematodes Exert Antimicrobial Activity and Benefit From Microbiota-Driven Support for Host Immune Regulation. Front Immuno/ 9, 2282, doi:10.3389/fimmu.2018.02282 (2018).

6. Steinfelder, S., Rausch, S., Michael, D., Kuhl, A. A. \& Hartmann, S. Intestinal helminth infection induces highly functional resident memory CD4(+) T cells in mice. Eur J Immuno/ 47, 353-363, doi:10.1002/eji.201646575 (2017).

7. Whelan, R. A. et al. A transgenic probiotic secreting a parasite immunomodulator for site-directed treatment of gut inflammation. Mol Ther 22, 1730-1740, doi:10.1038/mt.2014.125 (2014).

8. Ziegler, T. et al. A novel regulatory macrophage induced by a helminth molecule instructs IL-10 in CD4+ T cells and protects against mucosal inflammation. J Immuno/ 194, 1555-1564, doi:10.4049/jimmunol.1401217 (2015).

9. Grantham, B. D. \& Barrett, J. Amino acid catabolism in the nematodes Heligmosomoides polygyrus and Panagrellus redivivus. 2. Metabolism of the carbon skeleton. Parasitology 93 (Pt 3), 495-504, doi:10.1017/s0031182000081208 (1986).

10. Mossakowski, A. A. et al. Tracking CNS and systemic sources of oxidative stress during the course of chronic neuroinflammation. Acta Neuropatho/ 130, 799-814, doi:10.1007/s00401-015-1497-x (2015).

11. Radbruch, H. et al. Ongoing Oxidative Stress Causes Subclinical Neuronal Dysfunction in the Recovery Phase of EAE. Front Immunol 7, 92, doi:10.3389/fimmu.2016.00092 (2016).

12. Lindquist, R. L., Bayat-Sarmadi, J., Leben, R., Niesner, R. \& Hauser, A. E. NAD(P)H Oxidase Activity in the Small Intestine Is Predominantly Found in Enterocytes, Not Professional Phagocytes. Int $J$ Mol 
Sci 19, doi:10.3390/ijms19051365 (2018).

13. Denk, W., Strickler, J. H. \& Webb, W. W. Two-photon laser scanning fluorescence microscopy. Science 248, 73-76, doi:10.1126/science.2321027 (1990).

14. Dmitriev, R. I., Intes, X. \& Barroso, M. M. Luminescence lifetime imaging of three-dimensional biological objects. J Cell Sci 134, 1-17, doi:10.1242/jcs.254763 (2021).

15. Stringari, C. et al. Multicolor two-photon imaging of endogenous fluorophores in living tissues by wavelength mixing. Sci Rep 7, 3792, doi:10.1038/s41598-017-03359-8 (2017).

16. Steinmark, I. E. et al. Time-Resolved Fluorescence Anisotropy of a Molecular Rotor Resolves Microscopic Viscosity Parameters in Complex Environments. Small 16, e1907139, doi:10.1002/smll.201907139 (2020).

17. Stringari, C. et al. Phasor approach to fluorescence lifetime microscopy distinguishes different metabolic states of germ cells in a live tissue. Proc Natl Acad Sci U S A 108, 13582-13587, doi:10.1073/pnas.1108161108 (2011).

18. Corcoran, D. S. et al. DDR1 autophosphorylation is a result of aggregation into dense clusters. Sci Rep 9, 17104, doi:10.1038/s41598-019-53176-4 (2019).

19. Lagarto, J. L. et al. In vivo label-free optical monitoring of structural and metabolic remodeling of myocardium following infarction. Biomed Opt Express 10, 3506-3521, doi:10.1364/BOE.10.003506 (2019).

20. Sparks, H. et al. Heterogeneity in tumor chromatin-doxorubicin binding revealed by in vivo fluorescence lifetime imaging confocal endomicroscopy. Nat Commun 9, 2662, doi:10.1038/s41467018-04820-6 (2018).

21. Ranjit, S., Malacrida, L., Jameson, D. M. \& Gratton, E. Fit-free analysis of fluorescence lifetime imaging data using the phasor approach. Nat Protoc 13, 1979-2004, doi:10.1038/s41596-018-0026-5 (2018).

22. Ranjit, S., Malacrida, L., Stakic, M. \& Gratton, E. Determination of the metabolic index using the fluorescence lifetime of free and bound nicotinamide adenine dinucleotide using the phasor approach. J Biophotonics 12, e201900156, doi:10.1002/jbio.201900156 (2019).

23. Coban, O. et al. Effect of phosphorylation on EGFR dimer stability probed by single-molecule dynamics and FRET/FLIM. Biophys J 108, 1013-1026, doi:10.1016/j.bpj.2015.01.005 (2015).

24. Irshad, S. et al. RORgammat(+) Innate Lymphoid Cells Promote Lymph Node Metastasis of Breast Cancers. Cancer Res 77, 1083-1096, doi:10.1158/0008-5472.CAN-16-0598 (2017).

25. Nicholas, N. S. et al. PAK4 suppresses PDZ-RhoGEF activity to drive invadopodia maturation in melanoma cells. Oncotarget 7, 70881-70897, doi:10.18632/oncotarget.12282 (2016).

26. Lakowicz, J. R., Szmacinski, H., Nowaczyk, K. \& Johnson, M. L. Fluorescence lifetime imaging of calcium using Quin-2. Cell Calcium 13, 131-147, doi:10.1016/0143-4160(92)90041-p (1992).

27. Lakowicz, J. R., Szmacinski, H., Nowaczyk, K. \& Johnson, M. L. Fluorescence lifetime imaging of free and protein-bound NADH. Proc Natl Acad Sci U S A 89, 1271-1275, doi:10.1073/pnas.89.4.1271 
(1992).

28. Levitt, J. A. et al. Quantitative real-time imaging of intracellular FRET biosensor dynamics using rapid multi-beam confocal FLIM. Sci Rep 10, 5146, doi:10.1038/s41598-020-61478-1 (2020).

29. Datta, R., Gillette, A., Stefely, M. \& Skala, M. C. Recent innovations in fluorescence lifetime imaging microscopy for biology and medicine. J Biomed Opt 26, doi:10.1117/1.JB0.26.7.070603 (2021).

30. Poland, S. P. et al. A high speed multifocal multiphoton fluorescence lifetime imaging microscope for live-cell FRET imaging. Biomed Opt Express 6, 277-296, doi:10.1364/BOE.6.000277 (2015).

31. Dong, C. Y. et al. Fluorescence-lifetime imaging techniques for microscopy. Methods Cell Biol 72, 431464, doi:10.1016/s0091-679x(03)72021-4 (2003).

32. Ameer-Beg, S., Suhling, K. \& Kuimova, M. Special issue on fluorescence lifetime imaging (FLIM): from fundamentals to applications. Methods Appl Fluoresc 8, 040401, doi:10.1088/2050-6120/abad19 (2020).

33. Zhang, Y. et al. Automatic segmentation of intravital fluorescence microscopy images by K-means clustering of FLIM phasors. Opt Lett 44, 3928-3931, doi:10.1364/OL.44.003928 (2019).

34. Bremer, D., Leben, R., Mothes, R., Radbruch, H. \& Niesner, R. Method to Detect the Cellular Source of Over-Activated NADPH Oxidases Using NAD (P)H Fluorescence Lifetime Imaging. Curr Protoc Cytom 80, 952 51-59 52 14, doi:10.1002/cpcy.20 (2017).

35. Leben, R. et al. Phasor-Based Endogenous NAD(P)H Fluorescence Lifetime Imaging Unravels Specific Enzymatic Activity of Neutrophil Granulocytes Preceding NETosis. Int J Mol Sci 19, doi:10.3390/ijms19041018 (2018).

36. Digman, M. A., Caiolfa, V. R., Zamai, M. \& Gratton, E. The phasor approach to fluorescence lifetime imaging analysis. Biophys J 94, L14-16, doi:10.1529/biophysj.107.120154 (2008).

37. Qian, T. et al. Label-free imaging for quality control of cardiomyocyte differentiation. Nat Commun 12, 4580, doi:10.1038/s41467-021-24868-1 (2021).

38. Skala, M. et al. Heterogeneity of post-COVID impairment: interim analysis of a prospective study from Czechia. Virol J 18, 73, doi:10.1186/s12985-021-01546-8 (2021).

39. Skala, M. C. et al. In vivo multiphoton microscopy of NADH and FAD redox states, fluorescence lifetimes, and cellular morphology in precancerous epithelia. Proc Natl Acad Sci U S A 104, 1949419499, doi:10.1073/pnas.0708425104 (2007).

40. Skala, M. C. et al. Multiphoton microscopy of endogenous fluorescence differentiates normal, precancerous, and cancerous squamous epithelial tissues. Cancer Res 65, 1180-1186, doi:10.1158/0008-5472.CAN-04-3031 (2005).

41. Niesner, R. et al. Selective detection of NADPH oxidase in polymorphonuclear cells by means of NAD(P)H-based fluorescence lifetime imaging. J Biophys 2008, 602639, doi:10.1155/2008/602639 (2008).

42. Niesner, R., Peker, B., Schlusche, P. \& Gericke, K. H. Noniterative biexponential fluorescence lifetime imaging in the investigation of cellular metabolism by means of $N A D(P) H$ autofluorescence. 
Chemphyschem 5, 1141-1149, doi:10.1002/cphc.200400066 (2004).

43. Vishwasrao, H. D., Heikal, A. A., Kasischke, K. A. \& Webb, W. W. Conformational dependence of intracellular NADH on metabolic state revealed by associated fluorescence anisotropy. J Biol Chem 280, 25119-25126, doi:10.1074/jbc.M502475200 (2005).

44. Leben, R., Kohler, M., Radbruch, H., Hauser, A. E. \& Niesner, R. A. Systematic Enzyme Mapping of Cellular Metabolism by Phasor-Analyzed Label-Free NAD (P)H Fluorescence Lifetime Imaging. Int J Mol Sci 20, doi:10.3390/ijms20225565 (2019).

45. Mothes, R. et al. Teriflunomide Does Not Change Dynamics of Nadph Oxidase Activation and Neuronal Dysfunction During Neuroinflammation. Front Mol Biosci 7, 62, doi:10.3389/fmolb.2020.00062 (2020).

46. Dolowschiak, T. et al. Potentiation of epithelial innate host responses by intercellular communication. PLoS Pathog 6, e1001194, doi:10.1371/journal.ppat.1001194 (2010).

47. Schnoeller, C. et al. A helminth immunomodulator reduces allergic and inflammatory responses by induction of IL-10-producing macrophages. J Immuno/ 180, 4265-4272, doi:10.4049/jimmunol.180.6.4265 (2008).

48. Schubart, C. et al. Selective expression of constitutively activated STAT6 in intestinal epithelial cells promotes differentiation of secretory cells and protection against helminths. Mucosal Immunol 12, 413-424, doi:10.1038/s41385-018-0107-3 (2019).

49. Voehringer, D., Shinkai, K. \& Locksley, R. M. Type 2 immunity reflects orchestrated recruitment of cells committed to IL-4 production. Immunity 20, 267-277, doi:10.1016/s1074-7613(04)00026-3 (2004).

50. Voehringer, D. et al. Nippostrongylus brasiliensis: identification of intelectin-1 and -2 as Stat6dependent genes expressed in lung and intestine during infection. Exp Parasitol 116, 458-466, doi:10.1016/j.exppara.2007.02.015 (2007).

51. Hewitson, J. P. et al. Proteomic analysis of secretory products from the model gastrointestinal nematode Heligmosomoides polygyrus reveals dominance of venom allergen-like (VAL) proteins. $J$ Proteomics 74, 1573-1594, doi:10.1016/j.jprot.2011.06.002 (2011).

52. Ouzounov, D. G. et al. In vivo three-photon imaging of activity of GCaMP6-labeled neurons deep in intact mouse brain. Nat Methods 14, 388-390, doi:10.1038/nmeth.4183 (2017).

53. Gilad, A. \& Helmchen, F. Spatiotemporal refinement of signal flow through association cortex during learning. Nat Commun 11, 1744, doi:10.1038/s41467-020-15534-z (2020).

54. Helmchen, F. \& Denk, W. Deep tissue two-photon microscopy. Nat Methods 2, 932-940, doi:10.1038/nmeth818 (2005).

55. Helmchen, F., Denk, W. \& Kerr, J. N. Miniaturization of two-photon microscopy for imaging in freely moving animals. Cold Spring Harb Protoc 2013, 904-913, doi:10.1101/pdb.top078147 (2013).

56. Reimer, D. et al. B Cell Speed and B-FDC Contacts in Germinal Centers Determine Plasma Cell Output via Swiprosin-1/EFhd2. Cell Rep 32, 108030, doi:10.1016/j.celrep.2020.108030 (2020). 
57. Rakhymzhan, A. et al. Synergistic Strategy for Multicolor Two-photon Microscopy: Application to the Analysis of Germinal Center Reactions In Vivo. Sci Rep 7, 7101, doi:10.1038/s41598-017-07165-0 (2017).

58. Tas, J. M. et al. Visualizing antibody affinity maturation in germinal centers. Science 351, 1048-1054, doi:10.1126/science.aad3439 (2016).

59. Reismann, D. et al. Longitudinal intravital imaging of the femoral bone marrow reveals plasticity within marrow vasculature. Nat Commun 8, 2153, doi:10.1038/s41467-017-01538-9 (2017).

60. Bremer, D. et al. Longitudinal Intravital Imaging of the Retina Reveals Long-term Dynamics of Immune Infiltration and Its Effects on the Glial Network in Experimental Autoimmune Uveoretinitis, without Evident Signs of Neuronal Dysfunction in the Ganglion Cell Layer. Front Immuno/ 7, 642, doi:10.3389/fimmu.2016.00642 (2016).

61. Arwert, E. N. et al. A Unidirectional Transition from Migratory to Perivascular Macrophage Is Required for Tumor Cell Intravasation. Cell Rep 23, 1239-1248, doi:10.1016/j.celrep.2018.04.007 (2018).

62. Entenberg, D. et al. A permanent window for the murine lung enables high-resolution imaging of cancer metastasis. Nat Methods 15, 73-80, doi:10.1038/nmeth.4511 (2018).

63. Ginter, P. S. et al. Tumor Microenvironment of Metastasis (TMEM) Doorways Are Restricted to the Blood Vessel Endothelium in Both Primary Breast Cancers and Their Lymph Node Metastases. Cancers (Basel) 11, doi:10.3390/cancers11101507 (2019).

64. Heim, N. et al. Improved calcium imaging in transgenic mice expressing a troponin C-based biosensor. Nat Methods 4, 127-129, doi:10.1038/nmeth1009 (2007).

65. Ulbricht, C. et al. Intravital quantification reveals dynamic calcium concentration changes across $B$ cell differentiation stages. Elife 10, doi:10.7554/eLife.56020 (2021).

66. Strandmark, J. et al. Eosinophils are required to suppress Th2 responses in Peyer's patches during intestinal infection by nematodes. Mucosal Immunol 10, 661-672, doi:10.1038/mi.2016.93 (2017).

67. van der Hoeven, R., Cruz, M. R., Chavez, V. \& Garsin, D. A. Localization of the Dual Oxidase BLI-3 and Characterization of Its NADPH Oxidase Domain during Infection of Caenorhabditis elegans. PLoS One 10, e0124091, doi:10.1371/journal.pone.0124091 (2015).

\section{Figures}




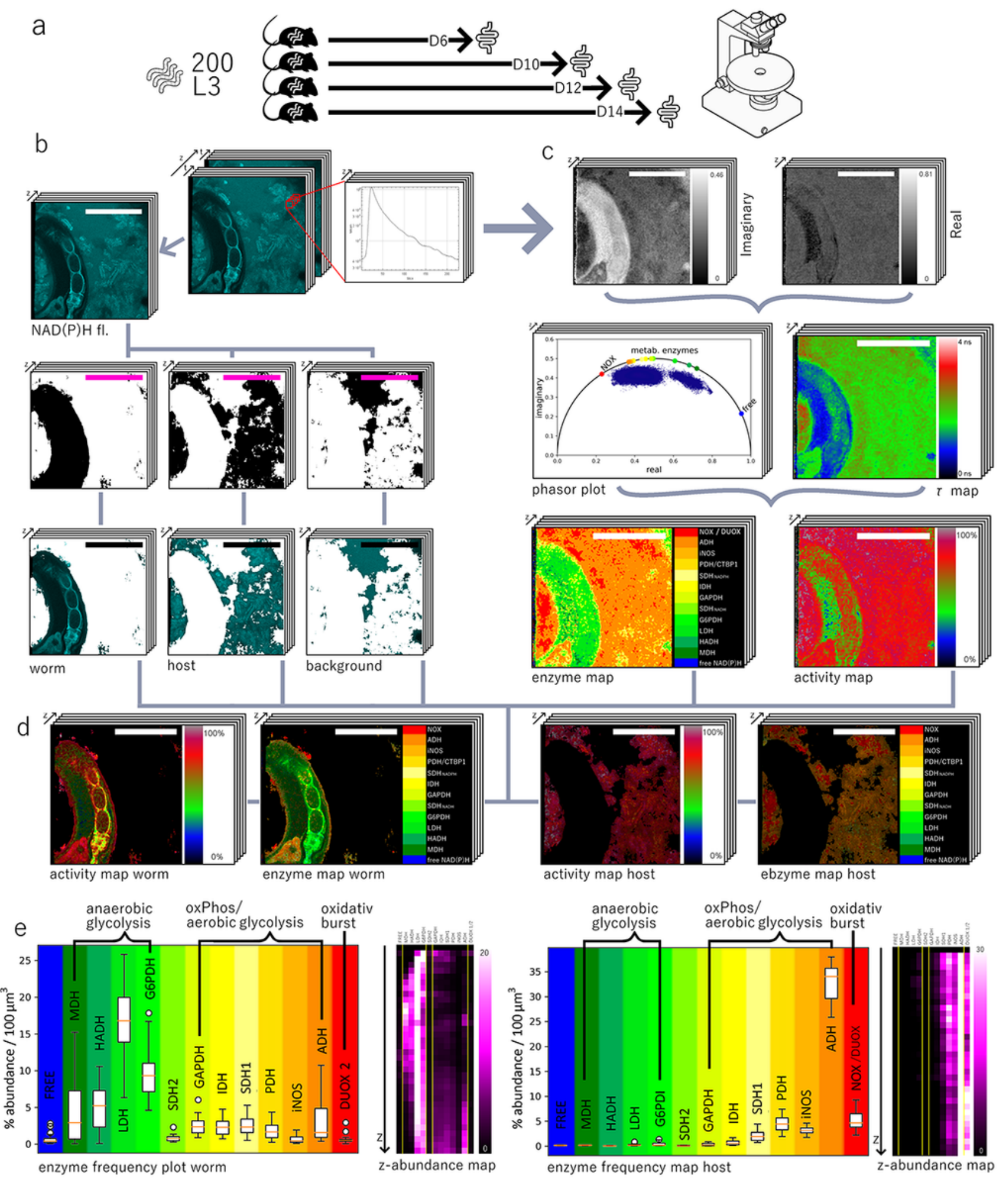

Figure 1

Principle of $\mathrm{NAD}(\mathrm{P}) \mathrm{H}$ fluorescence lifetime imaging of Heligmosomoides polygyrus in murine intestinal environment and data evaluation. (a) Schematics of experimental setup for infection and imaging. (b) Time-resolved $\mathrm{NAD}(\mathrm{P}) \mathrm{H}$ fluorescence images, i.e. for each pixel a fluorescence decay curve as shown is acquired, and $\mathrm{NAD}(\mathrm{P}) \mathrm{H}$ fluorescence sum images generation, typical results of $\mathrm{NAD}(\mathrm{P}) \mathrm{H}$ fluorescence sum image segmentation using the UNet pixel classification algorithm (llastik) and mask generation of 
various entities within the image, here parasite and host tissue. (c) Work-flow for phasor-based analysis of $\mathrm{NAD}(\mathrm{P}) \mathrm{H}$-FLIM data as acquired in (b), including the generation of maps showing the preferential $\mathrm{NAD}(\mathrm{P}) \mathrm{H}$-dependent enzymatic activity in each pixel (enzyme map) and maps showing the general $\mathrm{NAD}(\mathrm{P}) \mathrm{H}$ metabolic activity, i.e. activity maps. (d) Masked enzyme maps and activity maps for parasite and host intestinal tissue, respectively. All data are acquired in a three-dimensional manner, typically $500 \times 500 \times 100 \mu \mathrm{m}^{3}(505 \times 505 \times 51$ voxel). Scale bar for all images $=250 \mu \mathrm{m}$. (e) Corresponding pixel frequency graphs for the main $\mathrm{NAD}(\mathrm{P}) \mathrm{H}$-dependent enzymes in parasite (left side) and host tissue (right side), indicating the metabolic pathway that specific enzymatic activities are associated with. The purple graphs show the depth dependent frequency of enzyme activity for both parasite and host. 
a

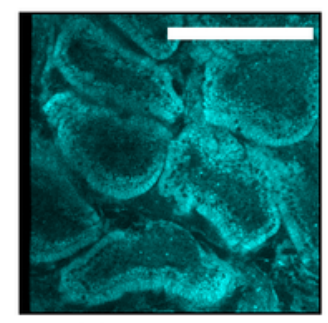

$\mathrm{NAD}(\mathrm{P}) \mathrm{H} \mathrm{fl}$

b
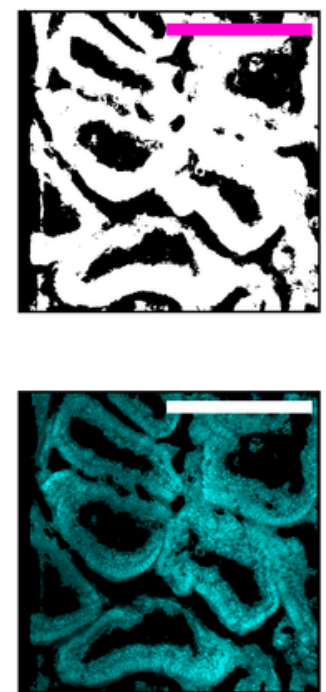

epithelium

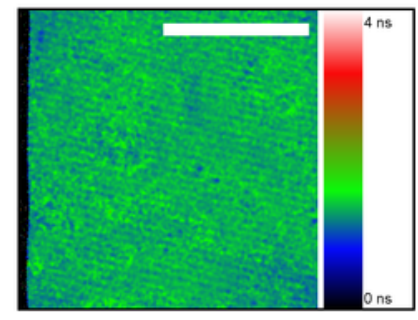

$\tau$ map
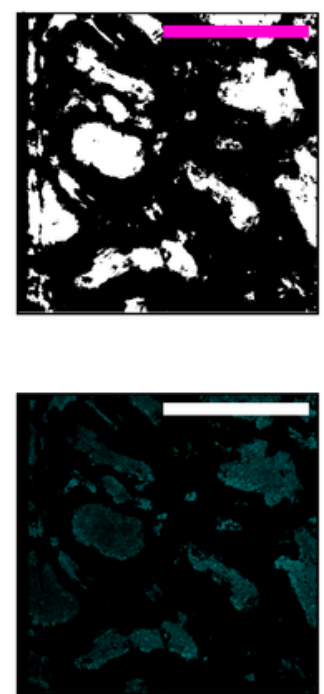

lamina propria

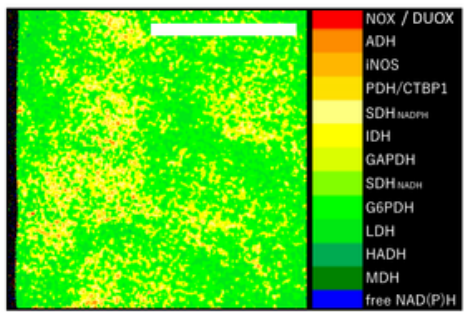

enzyme map

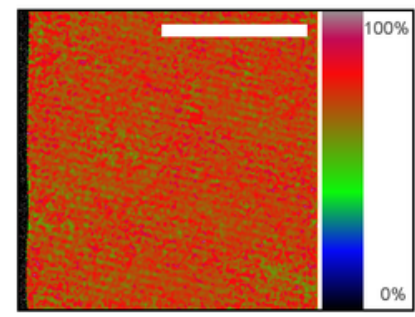

activity map
C
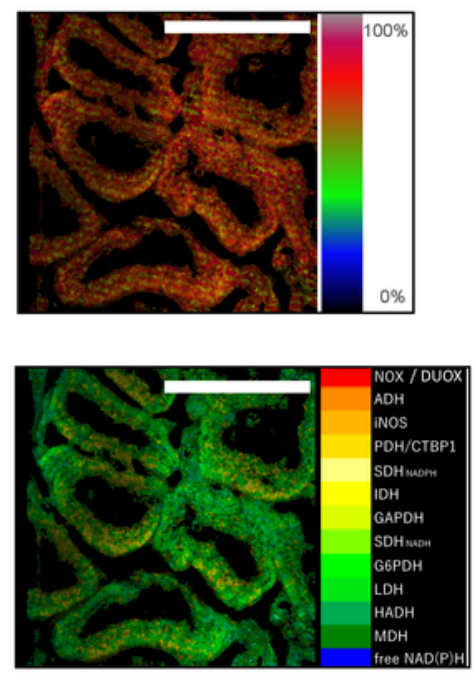

epithelium
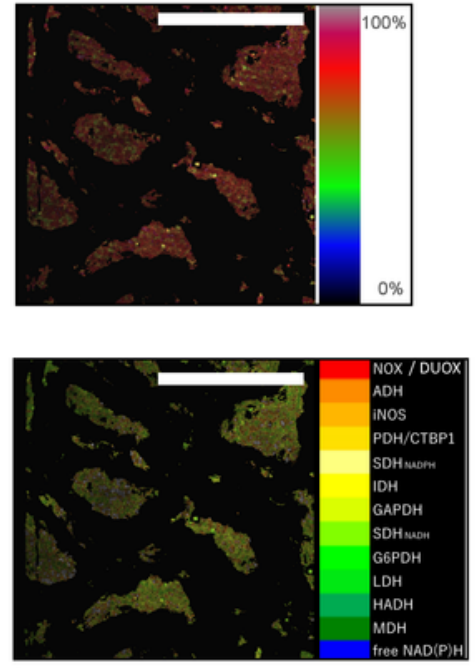

lamina propria
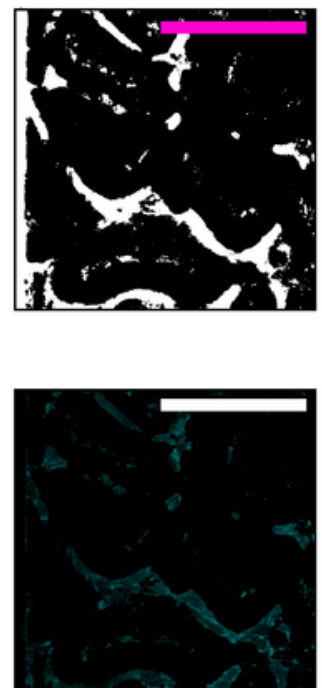

background
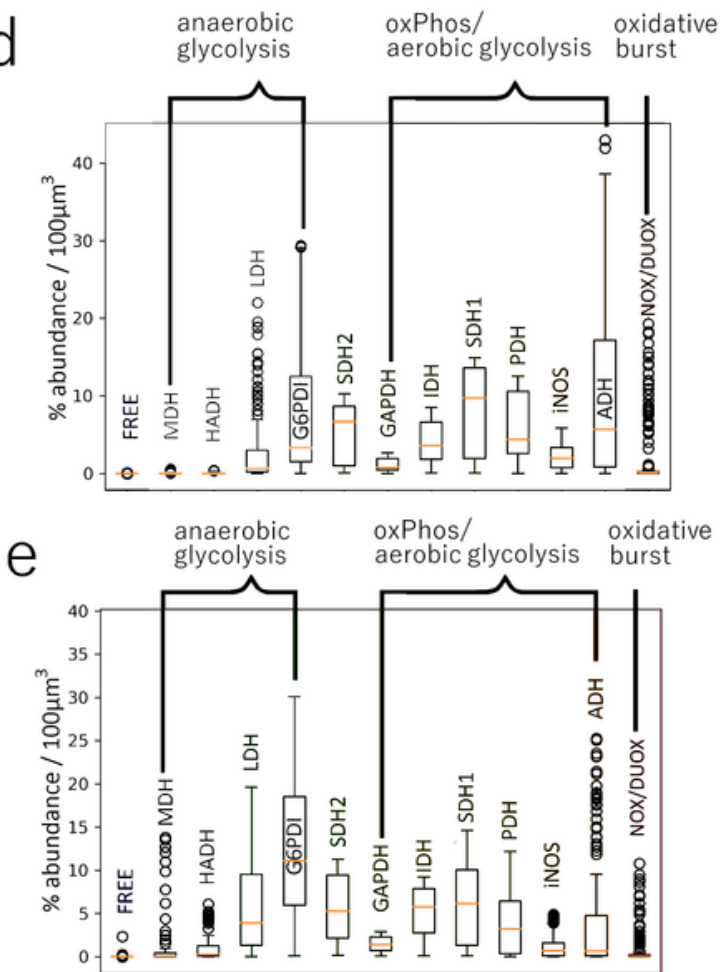

f

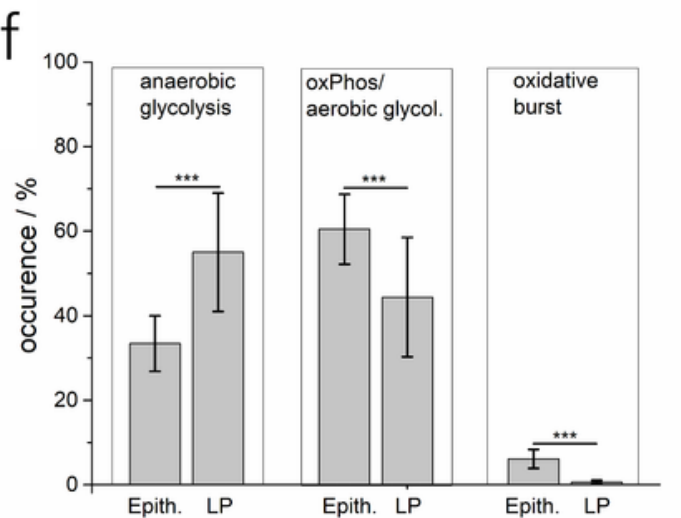

Figure 2

$\mathrm{NAD}(\mathrm{P}) \mathrm{H}$ fluorescence lifetime imaging of healthy explanted murine duodenum. (a) Representative $\mathrm{NAD}(\mathrm{P}) \mathrm{H}$ fluorescence sum image $\left(500 \times 500 \times 100 \mu \mathrm{m}^{3}\right)$, fluorescence lifetime image, enzyme map and activity map of healthy duodenum. (b) Segmentation of intestinal villi in epithelium and lamina propria using the same algorithm as described in Fig. 1. (c) Masked enzyme and activity maps for epithelium and lamina propria. (d) Frequency graph of enzymatic activity in epithelium (data from $n=6$ mice). (e) 
Frequency graph of enzymatic activity in lamina propria (data from $n=7$ mice). Scale bar $=250 \mu \mathrm{m}$. ( $\mathrm{f}$ ) Pixel frequencies of anaerobe glycolysis, oxidative phosphorylation (oxPhos) / aerobic glycolysis and oxidative burst, all given in percentage, for epithelial cells and lamina propria encompassing all mice in (d) and (e). Statistical analysis in (f) was performed using ANOVA test (ns $p>0.05, * p<0.05, * \star p<0.01$, $\star \star \star ~ p<0.001)$.

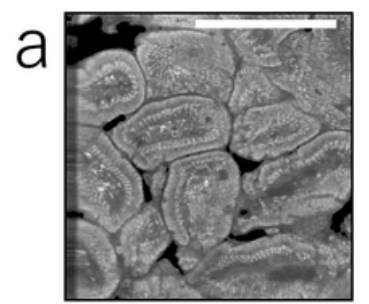

day 6
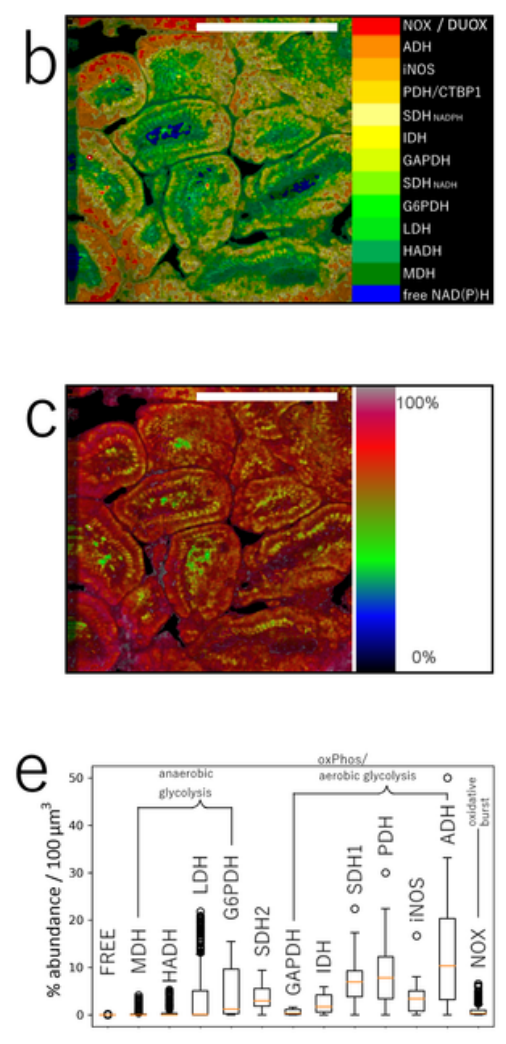
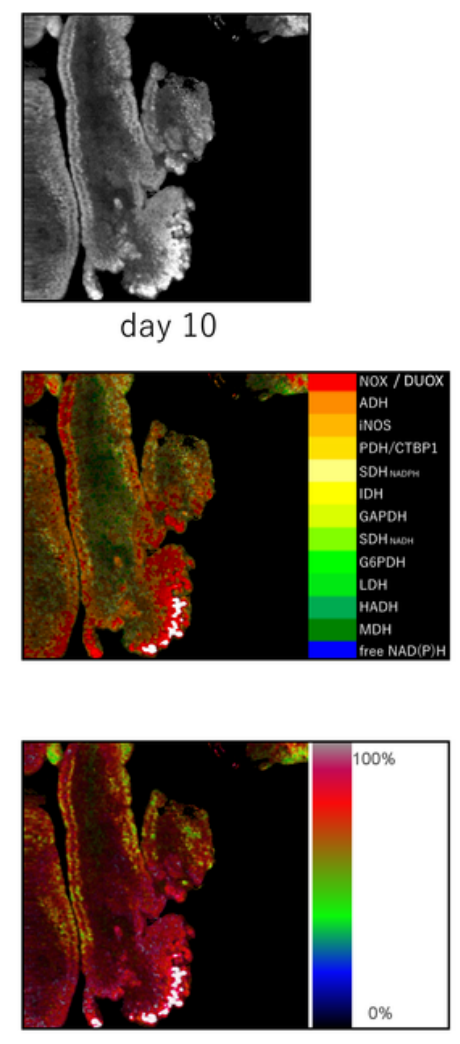

day 10
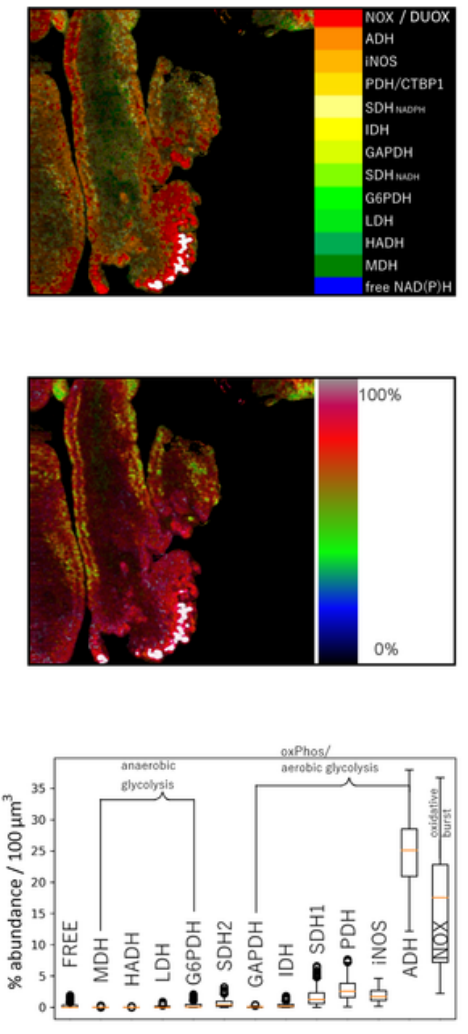

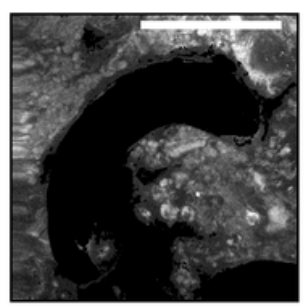

day 14
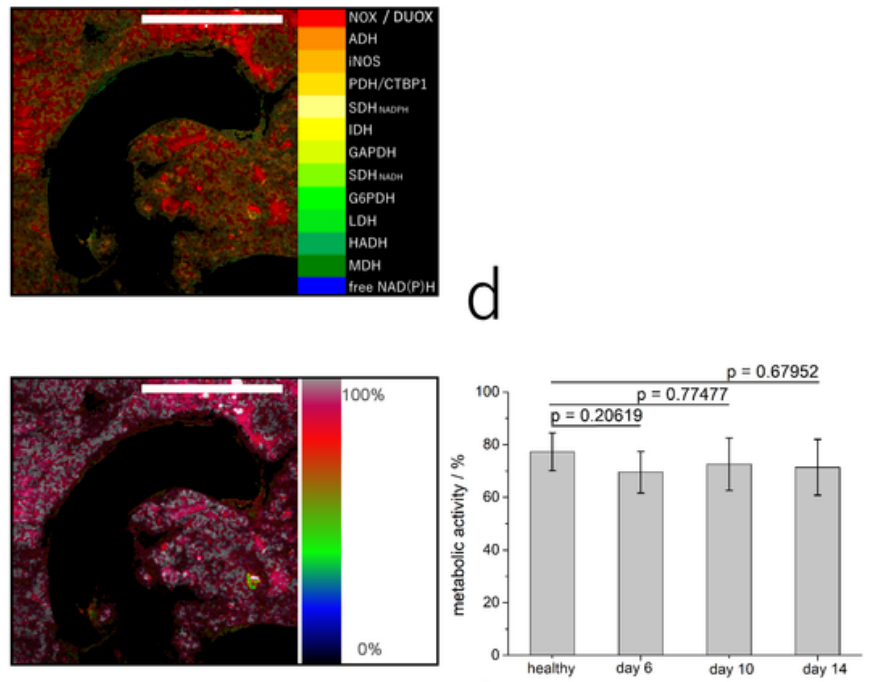

$f$
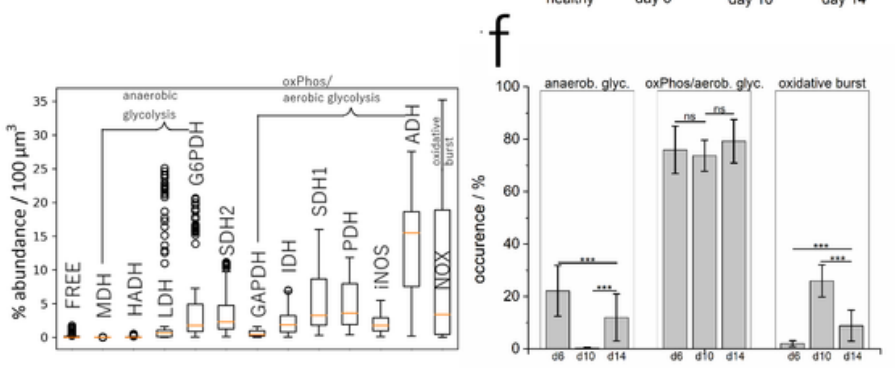

Figure 3

$\mathrm{NAD}(\mathrm{P}) \mathrm{H}$ fluorescence lifetime imaging of murine host duodenum from mice infected with $\mathrm{H}$. polygyrus. (a) Representative NAD(P)H fluorescence sum images $\left(500 \times 500 \times 100 \mu \mathrm{m}^{3}\right)$ of host tissue (villi) at day 6 , 10 and 14 after infection (masks generated by segmentation). Corresponding enzyme maps (b) and metabolic maps (c) of the NAD(P)H fluorescence sum images in (a). Scale bar for all images $=250 \mu \mathrm{m}$. (d) General metabolic activity of infected mice as compared to healthy mice $(n=2$ mice for day $6 ; n=3$ mice for day 10; $n=4$ mice for day 14 and $n=7$ healthy mice - Fig. 2). (e) Frequency graphs for the three time points during infection encompassing data from the same infected mice as in (d). (f) Pixel frequencies of anaerobe glycolysis, oxidative phosphorylation (oxPhos) / aerobic glycolysis and 
oxidative burst, all given in percentage, for villi tissue (including epithelium and lamina propria, without further segmentation) encompassing all mice in Fig. 3e and Fig. 2d,e. Statistical analysis in (d) and (f) was performed using ANOVA test with Bonferroni multi-column post-test ( $n s p>0.05,{ }^{*} p<0.05$, $* * p<$ $0.01, * \star \star p<0.001)$.

原

\section{Figure 4}

$\mathrm{NAD}(\mathrm{P}) \mathrm{H}$ fluorescence lifetime imaging of $\mathrm{H}$. polygyrus in intestinal environment. (a) Snapshots (500x500 $\mu \mathrm{m}$ images) of $\mathrm{H}$. polygyrus movement in the murine intestinal lumen. Endogenous fluorescence of parasite and host villi (green and blue), BODIPY1 fluorescence in mucus (red). .exc $=920$ $\mathrm{nm}$. (b) Representative NAD(P)H fluorescence sum image $\left(500 \times 500 \times 100 \mu \mathrm{m}^{3}\right)$ and segmentation of the worm compartments, differentiating between hydro-skeleton (low $\mathrm{NAD}(\mathrm{P}) \mathrm{H}$ fluorescence) and worm tissue (NAD(P)H-rich areas). (c) Enzyme and activity maps of the entire $\mathrm{NAD}(\mathrm{P}) \mathrm{H}$ signal (right panel) and masked enzyme and activity maps of the $\mathrm{NAD}(\mathrm{P}) \mathrm{H}$-rich worm tissue (left panel). (d) 3D reconstructions of the enzyme and activity maps shown in the left panel in (c). (e) Frequency graph of enzymatic activity in the entire parasite and depth-dependent frequency representation of enzymatic activity. Scale bar for all images $=250 \mu \mathrm{m}$.

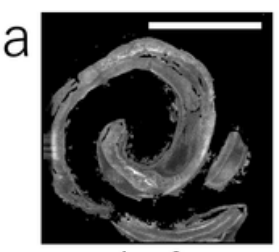

day 6
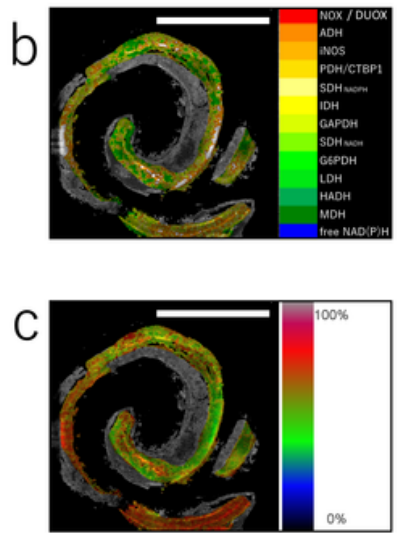

$\mathrm{e}$

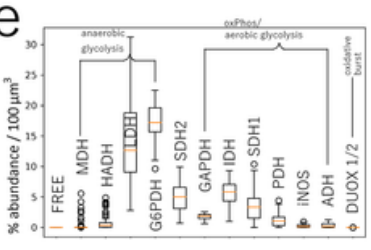

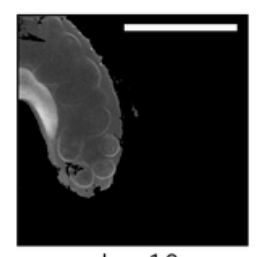

day 10
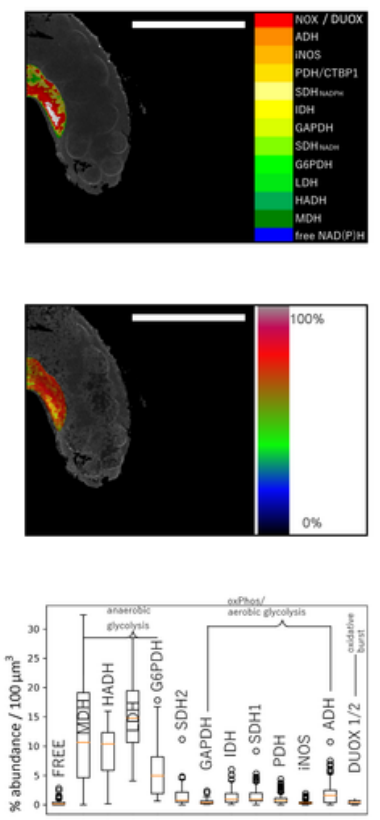

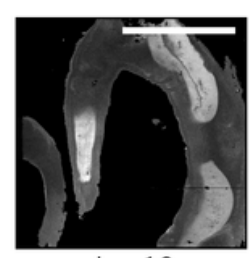

day 12
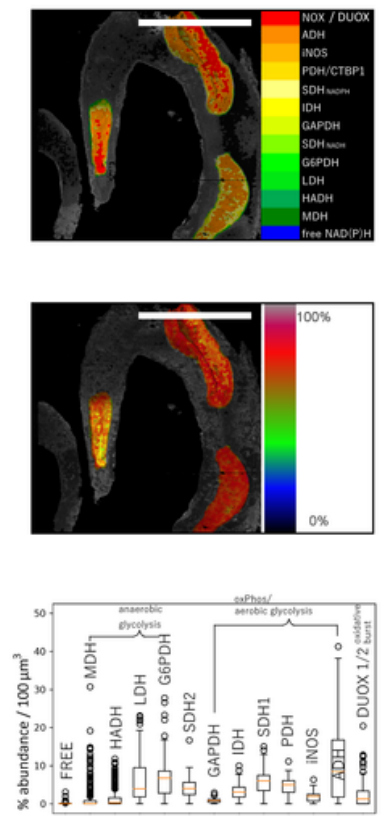

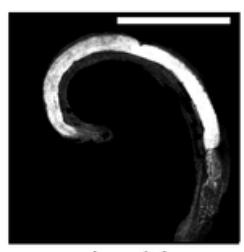

day 14
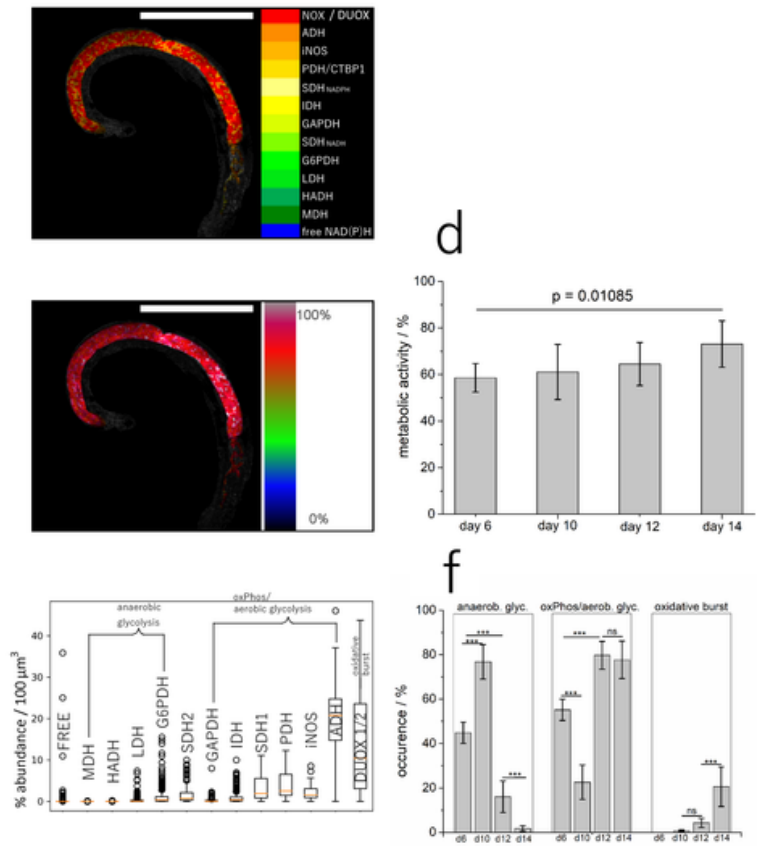

Figure 5 
$\mathrm{NAD}(\mathrm{P}) \mathrm{H}$ metabolism of $\mathrm{H}$. polygyrus over the course of infection (a) Representative masked NAD $(\mathrm{P}) \mathrm{H}$ fluorescence sum images $\left(500 \times 500 \times 100 \mu \mathrm{m}^{3}\right)$ of $\mathrm{NAD}(\mathrm{P}) \mathrm{H}$-rich parasite compartments at day $6,10,12$ and 14 after infection and corresponding enzyme activity maps (b) and activity maps (c). Scale bar $=250$ $\mu \mathrm{m}$. (d) Metabolic activity graph in parasites, at the same time points ( $n=2$ worms, day $6 ; n=5$ worms for day 10; $n=4$ worms, day 12; $n=5$ worms, day 14). (e) Corresponding frequency graphs of enzymatic activity for the same parasites as in (d). (f) Pixel frequencies of anaerobe glycolysis, oxidative phosphorylation (oxPhos) / aerobic glycolysis and oxidative burst, all given in percentage, encompassing all nematodes in (d). Statistical analysis in (f) was performed using ANOVA test with Bonferroni multicolumn post-test (ns $p>0.05, * p<0.05, * \star p<0.01, * \star \star p<0.001)$.

\section{Supplementary Files}

This is a list of supplementary files associated with this preprint. Click to download.

- Movie1.avi

- Movie2.avi

- Supplementalinformation.docx

- SupplementalFigure1.png 